\title{
X-Ray Studies of Surfactant Ordering and Interfacial Phases at the Water-Oil Interface
}

\author{
Sai Venkatesh Pingali \\ Department of Physics, University of Illinois at Chicago, Chicago, Illinois, USA
}

Takanori Takiue

Department of Chemistry, Faculty of Sciences, Kyushu University, Fukuoka, Japan

\section{Guangming Luo}

Department of Physics, University of Illinois at Chicago, Chicago, Illinois, USA

\author{
Aleksey M. Tikhonov \\ Center for Advanced Radiation Sources, University of Chicago, and Brookhaven National Laboratory, \\ National Synchrotron Light Source, Upton, New York, USA
}

\section{Norihiro Ikeda}

Department of Environment Science, Faculty of Human Environmental Science, Fukuoka Women's University, Fukuoka, Japan

\section{Makoto Aratono}

Department of Chemistry, Faculty of Sciences, Kyushu University, Fukuoka, Japan

\author{
Mark L. Schlossman \\ Departments of Physics and Chemistry, University of Illinois at Chicago, Chicago, Illinois, USA
}

\begin{abstract}
$X$-ray reflectivity studies of surfactants at the water-oil interface yield structural information with sub-nanometer resolution. In this presentation, we reviewed recent $X$-ray reflectivity measurements of the interface between water and a hexane solution of the hydrocarbon alkanol $\mathrm{CH}_{3}\left(\mathrm{CH}_{2}\right)_{19} \mathrm{OH}$ and fluorocarbon alkanol $\mathrm{CF}_{3}\left(\mathrm{CF}_{2}\right)_{7}\left(\mathrm{CH}_{2}\right)_{2} \mathrm{OH}$. The mixed system exhibits three monolayer phases, two of which are similar to single surfactant phases. A transition from a liquid monolayer to a solid monolayer occurs with increasing temperature. This unusual phase transition and the qualitative features of the phase diagram are predicted by an appropriate superposition of the behavior of the two single surfactant systems.
\end{abstract}

Keywords X-ray reflectivity, surfactants, water-oil interface

\section{INTRODUCTION}

Many phenomena are determined by surfactant adsorption at liquid-liquid interfaces. Structural information on the surfactant conformation, ordering, and phase behavior at this interface is scarce, primarily due to the lack of appropriate structural probes. Recently, we introduced X-ray reflectivity as a probe of surfactant ordering at the liquid-liquid interface. ${ }^{[1]}$ The primary structural information determined by X-ray reflectivity

Received 10 October 2005; Accepted 16 October 2005.

Address correspondence to Mark L. Schlossman, Department of Physics, University of Illinois at Chicago, 845 West Taylor St., Chicago, IL 60607, USA. E-mail: schloss@uic.edu is the variation of electron density with interfacial depth. At the water-oil interface, this variation is determined with sub-nanometer, $\sim 5 \AA$ A, spatial resolution along the interfacial normal. As an example, Figure 1 illustrates the electron density profile for a triacontanol $\left(\mathrm{CH}_{3}\left(\mathrm{CH}_{2}\right)_{29} \mathrm{OH}\right)$ monolayer at the waterhexane interface. ${ }^{[2]}$ Variation of the electron density in the region of the tail group can be interpreted as a variation of the disorder along the length of the alkyl chain. As we have shown, the lower electron density in the terminal region of the chain corresponds to a disorder similar to that found in bulk alkane liquids just above their melting point. Closer to the head group the chains are more ordered. The ability of X-ray reflectivity to probe interfacial structural information on the 


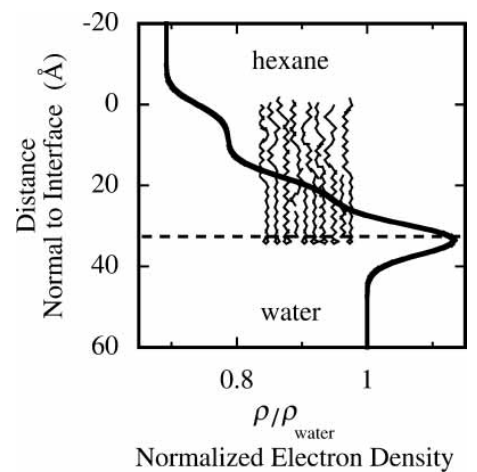

FIG. 1. Electron density as a function of depth through the water-hexane interface of a monolayer of triacontanol as measured by X-ray reflectivity. The peak indicates the extra electron density due to the head groups. The values of electron density in the tail group region can be interpreted to yield chain ordering as described in the text. The electron densities are normalized to the value for water (e.g., $0.3337 \mathrm{e}^{-} / \AA^{3}$ at $20^{\circ} \mathrm{C}$ ).

molecular level allows for the study of surfactant conformations as well as interfacial phases and phase transitions.

Figure 2 is a schematic of the sample cell. A hexane solution of surfactants is poured on top of the water surface to form the water-hexane interface. The thermodynamic state of this system is determined by the temperature, bulk pressure, and bulk compositions. Under appropriate conditions the surfactants will adsorb onto the water-hexane interface to form a monolayer. They can also desorb from the interface, thereby returning to the bulk hexane, which acts as a reservoir of surfactants. As a result of this exchange, the interfacial surfactant layers are in equilibrium, unlike the metastable monolayers often formed as Langmuir monolayers at the water-vapor interface. ${ }^{[3]}$ As discussed in more detail below, $X$-rays that probe the interface are transmitted through the upper, bulk hexane phase, then reflected off the water-hexane interface.

\section{MATERIALS}

The mixed surfactant system consists of two surfactants, 1-eicosanol $\left(\mathrm{CH}_{3}\left(\mathrm{CH}_{2}\right)_{19} \mathrm{OH}\right.$, denoted $\mathrm{C}_{20} \mathrm{OH}$, purchased

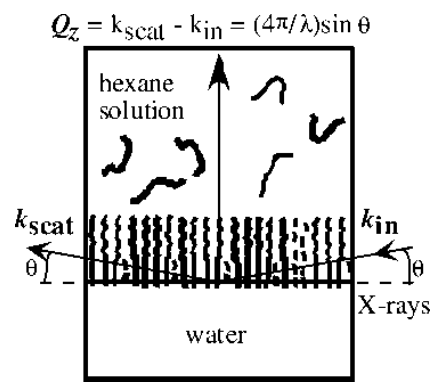

FIG. 2. X-ray specular reflectivity kinematics from the interface between water and a hexane solution of surfactants with equal incident and reflection angles $\theta$. The wave vector transfer, $Q_{z}=(4 \pi / \lambda) \sin \theta$, is normal to the interface. The size of the molecules is greatly exaggerated. The $\mathrm{X}$-rays pass through the upper hexane solution, then reflect off the surfactant monolayer at the interface. from Sigma Ltd.) and 1,1,2,2-tetrahydroheptadecafluorodecanol $\left(\mathrm{CF}_{3}\left(\mathrm{CF}_{2}\right)_{7}\left(\mathrm{CH}_{2}\right)_{2} \mathrm{OH}\right.$, denoted $\mathrm{FC}_{10} \mathrm{OH}$, purchased from Lancaster Ltd.). These surfactants are purified by several crystallizations in purified hexane. The hexane is purified by passing it six times through a column of basic alumina. Purified water is from a Barnstead Nanopure system. The purity of hexane and water is checked by the value and stability of the water-hexane interfacial tension; the purity of the surfactants is checked by gas-liquid chromatography. The study of these two surfactants is interesting due to the inherent flexibility of the $\mathrm{C}_{20} \mathrm{OH}$ chains and rigidity of the $\mathrm{FC}_{10} \mathrm{OH}$ chains, as well as their very different intermolecular interactions.

\section{EXPERIMENTAL METHOD}

$\mathrm{X}$-ray reflectivity was measured at beamline X19C at the National Synchrotron Light Source (Brookhaven National Laboratory) with a liquid surface instrument and measurement techniques described in detail elsewhere. ${ }^{[4-6]}$ The kinematics of specular reflectivity is illustrated in Figure 2. The reflectivity data are measured as a function of wave vector transfer normal to the interface, $Q_{z}=(4 \pi / \lambda) \sin \theta$ (the in-plane wave vector components $Q_{x}=Q_{y}=0$ where $\lambda=0.825 \pm 0.002 \AA$ is the $\mathrm{X}$-ray wavelength and $\theta$ is the angle of reflection). Therefore, specular reflection probes structure normal to the interface, but averaged over the in-plane region of the interface.

X-ray reflectivity $R\left(Q_{z}\right)$ from the water-hexane interface can be analyzed to yield the electron density profile by use of the first Born approximation, written as ${ }^{[7]}$

$$
\frac{R\left(Q_{z}\right)}{R_{F}\left(Q_{z}\right)} \approx\left|\frac{1}{\rho_{W}-\rho_{H}} \int d z \frac{d\left\langle\rho_{e}(z)\right\rangle_{x y}}{d z} \exp \left(i Q_{z} z\right)\right|^{2}
$$

where $z$ is in the normal direction, $\left\langle\rho_{e}(z)\right\rangle_{x y}$ is the electron density profile averaged over the surface area of the interface (in the x-y plane), $\rho_{W}$ and $\rho_{H}$ are the electron densities of bulk water and hexane, respectively (e.g., $\rho_{W}=0.3337 \mathrm{e}^{-} /$ $\AA^{3}$ and $\rho_{H}=0.230 \mathrm{e}^{-} / \AA^{3}$ at $T=20^{\circ} \mathrm{C}$ ), the Fresnel reflectivity $R_{\mathrm{F}}$ is calculated for an ideal interface whose electron density changes abruptly from the value of one bulk phase to the other, and is expressed as

$$
R_{F}\left(Q_{z}\right) \approx\left|\frac{Q_{z}-Q_{z}^{T}}{Q_{z}+Q_{z}^{T}}\right|^{2}
$$

where $Q_{z}^{T}=\left(Q_{z}^{2}-Q_{c}^{2}\right)^{1 / 2}$ is the $z$-component of the wave vector transfer with respect to the lower phase. Total reflection of X-rays from the lower phase occurs for $Q_{z} \leq Q_{c}$ where the critical wave vector transfer is $Q_{c}=4\left(\pi r_{e}\left(\rho_{W}-\rho_{H}\right)\right)^{1 / 2}$ $\approx 0.012 \AA^{-1}$ ( $r_{\mathrm{e}}$ is the classical electron radius).

Equation (1) indicates that the reflectivity probes the gradient of the electron density along the interfacial depth, averaged over the $x-y$ plane. This explains the sensitivity of the technique to surface or interfacial structure. Upon passing through the bulk hexane solution, the X-rays encounter regions of varying 
electron density and are scattered from these bulk variations. That scattering does not produce a reflected X-ray beam because the bulk variations, once averaged over the $\mathrm{x}-\mathrm{y}$ plane, do not produce a nonzero gradient. When the X-rays encounter, say, the top of the monolayer (see Figure 2), their scattering produces a reflected beam because there is a nonzero average electron density gradient due to the change in chemical composition that occurs at the top of the monolayer. Similarly, when $\mathrm{X}$-rays encounter the bottom of the monolayer (this particular ray is shown in Figure 2), the average electron density gradient again changes, producing another reflected X-ray beam. Likewise, any electron density gradients present at different depths within the monolayer will also contribute to the reflected beam. Each of these reflections, from different depths within the monolayer, will add coherently to produce an interference pattern. In this sense, the monolayer can be considered as an X-ray interferometer. The shape and intensity of the interference fringes present in the reflected X-rays are analyzed to yield the electron density variation with depth through the interface.

\section{SINGLE SURFACTANT SYSTEMS}

\section{X-Ray Reflectivity}

An X-ray reflectivity study of a single surfactant at the water-hexane interface, $\mathrm{FC}_{10} \mathrm{OH}$, is depicted in Figure $3{ }^{[5,8]}$ When compared to the reflectivity from a pure water-hexane interface (see Figure 3), the reflection from the water-hexane $\left(\mathrm{FC}_{10} \mathrm{OH}\right)$ interface is greater. The increase in reflectivity over that of the pure interface is due to constructive interference of the X-rays reflected from the top and bottom of the monolayer. The reflection from the water-hexane $\left(\mathrm{FC}_{10} \mathrm{OH}\right)$ interface is essentially the first interference fringe in an interference pattern. More fringes would be visible if these measurements could be carried out at higher reflection angles (i.e., larger wave vector transfer $Q_{z}$ ).

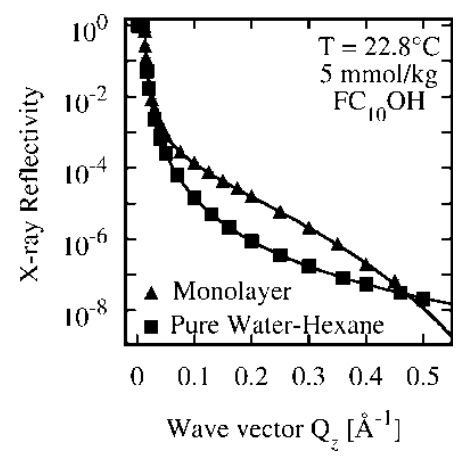

FIG. 3. Comparison of the X-ray reflectivity curves between an $\mathrm{FC}_{10} \mathrm{OH}$ monolayer at water-hexane interface $(\boldsymbol{\Lambda})$ and the pure water-hexane interface (ם). The X-ray reflectivity data for the monolayer was taken for a molal concentration of $5.0 \mathrm{mmol} / \mathrm{kg}$ of $\mathrm{FC}_{10} \mathrm{OH}$ in hexane at $\mathrm{T}=22.8^{\circ} \mathrm{C}$.
The reflectivity from the water-hexane $\left(\mathrm{FC}_{10} \mathrm{OH}\right)$ interface is analyzed with a single slab model. As depicted in Figure 4, the name of this model refers to a single slab (or layer) of uniform electron density that is sandwiched between bulk water and bulk hexane. This provides a low resolution model of the surfactant layer that does not include individual atoms, but allows the monolayer thickness and average electron density to be determined. The fact that only one slab is required to analyze these data indicates that no other significant electron density gradients are present in the monolayer (within the spatial resolution of these measurements, $\sim 5 \AA$ ). The smooth curve in Figure 4 is a smeared version of the slab model in which the density at the borders of the slab are smeared to provide a smooth crossover between the slab and the two bulk phases. This is a physical effect due to thermal fluctuations of the water-hexane interface, known as capillary waves, that result in a time-averaged smearing of the profile as measured by X-ray reflectivity. The smearing is characterized by an interfacial roughness (or width) $\sigma$ that can be calculated from capillary wave theory (and a measured value of the interfacial tension). Alternatively, the roughness can be determined by fitting the X-ray reflectivity.

The thickness of the monolayer determines the period of oscillation of the reflectivity interference fringe. As shown in Figure 4, the thickness of the monolayer as determined by $\mathrm{X}$-ray reflectivity is within a standard deviation of the calculated length of the fluorinated part of the molecule $\left(\mathrm{L}_{\text {fit }}=10 \pm 1 \AA ; \quad \mathrm{L}_{\text {calcuated }}=10.1 \AA\right)$. Due to the large electron density of the fluorinated chain, the near match of the head group electron density with the density of the water, and the range of wave vector transfer for this measurement, it can be shown that these measurements are not sensitive to the presence of the head group. Note that our other measurements of hydrocarbon surfactants are sensitive to the presence of the head group. This analysis indicates that

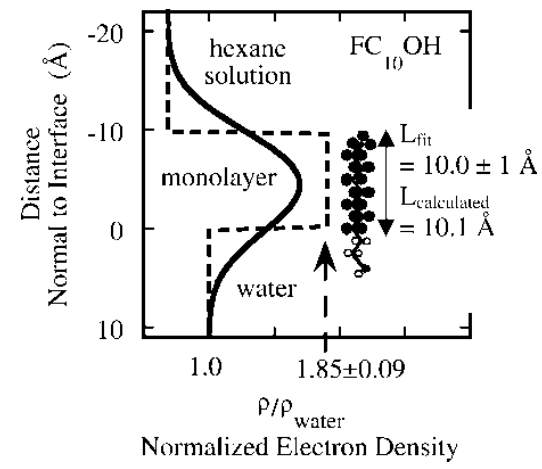

FIG. 4. Electron density profile of the $\mathrm{FC}_{10} \mathrm{OH}$ monolayer at the waterhexane interface obtained from X-ray reflectivity (see Figure 3) for two cases: dashed line, without thermal capillary wave fluctuations of the interface, and solid line, with thermal fluctuations of the interfaces. $\mathrm{FC}_{10} \mathrm{OH}$ molecular cartoon is approximately to scale. 
the $\mathrm{FC}_{10} \mathrm{OH}$ molecules are oriented nearly perpendicular to the water-hexane interface.

Analysis of the reflectivity also reveals that the average electron density in the $\mathrm{FC}_{10} \mathrm{OH}$ monolayer is $0.617 \pm$ $0.030 \mathrm{e}^{-} / \AA^{3}$ (or a value of $1.85 \pm 0.09$ times the electron density of water). This electron density corresponds to a mass density of $2.13 \mathrm{~g} / \mathrm{cm}^{3}$, which compares favorably to the density $\left(2.16 \mathrm{~g} / \mathrm{cm}^{3}\right)$ of the solid rotator phase of bulk fluoroalkanes. ${ }^{[9]}$ The electron density of hexane $\left(0.230 \mathrm{e}^{-} / \AA^{3}\right)$ is much lower than the electron density of the monolayer. If a significant amount of hexane mixed into the monolayer (say, a few percent), the electron density of the monolayer would be measurably lower. As determined by these $\mathrm{X}$-ray reflectivity measurements, the interface consists of a close-packed monolayer of $\mathrm{FC}_{10} \mathrm{OH}$ molecules oriented nearly perpendicular to the interface.

It is common to present a normalized version of X-ray reflectivity in which the data are divided by the calculated Fresnel reflectivity, given by Equation (2). A set of normalized $\mathrm{X}$-ray reflectivity curves for a range of temperatures measured from the water-hexane $\left(\mathrm{FC}_{10} \mathrm{OH}\right)$ system is shown in Figure $5 .^{[3,8]}$ Analysis of data at the lowest temperature, $22.8^{\circ} \mathrm{C}$, has just been discussed and corresponds to a fully covered interface of $\mathrm{FC}_{10} \mathrm{OH}$ molecules. The peak in the reflectivity is at the same position for all temperatures. This indicates that the monolayer thickness is independent of temperature. The decrease in peak amplitude for increasing temperature reveals a reduction in the number of molecules at the interface. Figure 6(a) illustrates three different structures of a lower density monolayer that are possible: (i) a low density of domains that do not fully cover the interface, but each domain consists of close-packed $\mathrm{FC}_{10} \mathrm{OH}$ molecules, (ii) a homogeneously covered interface of $\mathrm{FC}_{10} \mathrm{OH}$ molecules with the same orientation as in the fully packed monolayer, but with larger spacing between the molecules, and (iii) molecules that are oriented and spaced differently than in the fully packed monolayer. For option (iii) the functional form of the electron density with interfacial depth is different from options (i) and (ii), both of which have the same functional form. Option



FIG. 5. Normalized reflectivity $\left(R / R_{\mathrm{F}}\right)$ vs. wave vector $Q_{z}$ for the single surfactant $\mathrm{FC}_{10} \mathrm{OH}$ system at six different temperatures: (O) $22.8^{\circ} \mathrm{C}$, (घ) $27.6^{\circ} \mathrm{C},(\diamond) 30.1^{\circ} \mathrm{C},(\Delta) 36.4^{\circ} \mathrm{C},(\bullet) 45.9^{\circ} \mathrm{C},(\nabla) 56.5^{\circ} \mathrm{C}$.

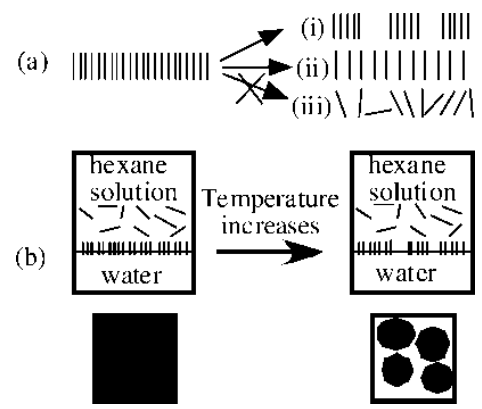

FIG. 6. Phase transition picture of the single surfactant $\mathrm{FC}_{10} \mathrm{OH}$ system (a) Three different options for the transition of the homogeneously covered monolayer of $\mathrm{FC}_{10} \mathrm{OH}$ molecules are: (i) a low density of domains that do not fully cover the interface, but each of which consists of close-packed $\mathrm{FC}_{10} \mathrm{OH}$ molecules, (ii) a homogeneously covered interface of $\mathrm{FC}_{10} \mathrm{OH}$ molecules with the same orientation as in the fully packed monolayer, but with larger spacing between the molecules, and (iii) molecules that are oriented and spaced differently than in the fully packed monolayer. Option (i) is correct; see discussion in text. (b) Summary of the phase transition from a full monolayer to a low density of monolayer domains with increasing temperature.

(iii) is excluded by our data because the shape of the reflectivity curve is unchanged with temperature, only the amplitude changes. This indicates that the functional form of the electron density profile is unchanged with temperature. Both options (i) and (ii) are possible explanations of our reflectivity data. However, Brewster angle microscopy (BAM) images of single surfactant $\mathrm{C}_{18} \mathrm{OH}$ and $\mathrm{FC}_{12} \mathrm{OH}$ at the water-hexane interface $^{[10]}$ and our $\mathrm{X}$-ray off-specular diffuse scattering studies of the single surfactant $\mathrm{FC}_{12} \mathrm{OH}$ system at the waterhexane interface directly probe the presence of domains. ${ }^{[3]}$ As discussed elsewhere, X-ray coherence effects on the reflectivity also require the presence of domains in the waterhexane $\left(\mathrm{FC}_{10} \mathrm{OH}\right)$ system. ${ }^{[11]}$ Therefore, the phase transition of the $\mathrm{FC}_{10} \mathrm{OH}$ monolayer with increasing temperature is from a full $\mathrm{FC}_{10} \mathrm{OH}$ monolayer to a lower density of $\mathrm{FC}_{10} \mathrm{OH}$ domains, as illustrated in Figure 6(b).

\section{Interfacial Domain Coverage}

Figure 7 summarizes the behavior of single surfactant $\mathrm{C}_{20} \mathrm{OH}$ and $\mathrm{FC}_{10} \mathrm{OH}$ systems at the water-hexane interface $^{[4,11]}$ The interfacial tension for the pure water-hexane interface has a small negative slope with temperature whereas the water-hexane interface with surfactants has one kink or change of slope. The interfacial excess entropy per unit area is defined by the slope of the tension $\gamma(\mathrm{T})$ curve,

$$
S_{a}^{\sigma}=-\left(\frac{\partial \gamma}{\partial T}\right)_{p, c}
$$

The negative slope of the tension curve for the pure waterhexane interface indicates a positive excess entropy, so water and hexane molecules have a greater entropy at the interface than in the bulk. Similarly, the large positive slope in the 


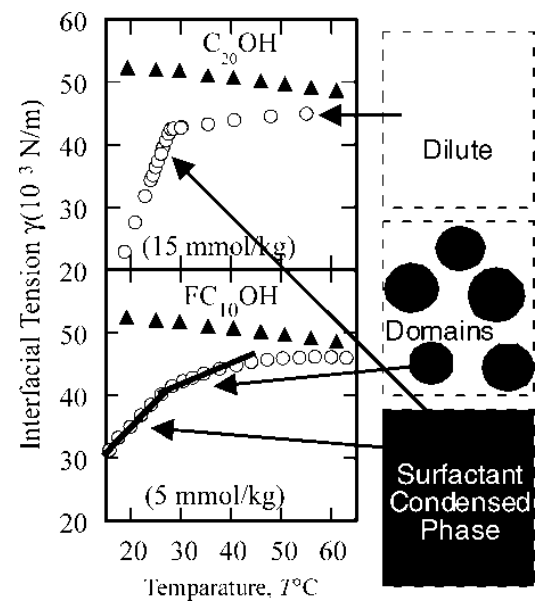

FIG. 7. Interfacial tension vs. temperature phase diagrams of the single surfactant systems. $\mathrm{C}_{20} \mathrm{OH}$ and $\mathrm{FC}_{10} \mathrm{OH}$ : (ム) Pure water-hexane interface, (O) Interface between water and hexane solution of surfactants. (Top left) Single surfactant $\mathrm{C}_{20} \mathrm{OH}$ system: a phase transition of condensed phase to dilute. (Bottom left) Single surfactant $\mathrm{FC}_{10} \mathrm{OH}$ system: a phase transition of a condensed phase to a low density of domains.

tension of the water-hexane $\left(\mathrm{C}_{20} \mathrm{OH}\right)$ system indicates a much smaller entropy of molecules at the interface than in the bulk. This can be interpreted as indicating the presence of a condensed monolayer of $\mathrm{C}_{20} \mathrm{OH}$ at the interface. Above the transition the excess interfacial entropy is still negative, although much smaller in magnitude. Therefore, with increasing temperature the interface undergoes an order-disorder transition. Note that the molecular length scale structure is obtained only from the X-ray reflectivity measurements.

Figure 7 illustrates, in cartoon format, the qualitative features determined from a number of studies of surfactants at the water-hexane interface. ${ }^{[3,4,8,11]}$ Below the transition temperature, the interface is fully covered by a condensed phase of surfactants. From the density of the monolayer as determined by our reflectivity measurements, we know that the $\mathrm{C}_{20} \mathrm{OH}$ surfactants form a liquid monolayer phase while the $\mathrm{FC}_{10} \mathrm{OH}$ surfactants form a solid monolayer phase. ${ }^{[11]}$ For some surfactants, as discussed elsewhere, the low-temperature phase can be a high-density domain phase that does not fully cover the interface. ${ }^{[4]}$ Above the transition temperature, the interface is covered either by a dilute gas monolayer phase of surfactants (as for $\mathrm{C}_{20} \mathrm{OH}$ ) or by a low-density domain monolayer phase (as for $\mathrm{FC}_{10} \mathrm{OH}$ ).

Figure 8 shows the domain coverage, $\mathrm{C}$ (i.e., the fraction of interface covered by surfactant domains), as a function of temperature. Desorption of the $\mathrm{C}_{20} \mathrm{OH}$ molecules from the interface with increasing temperature is a complete as well as an abrupt process. Desorption of the $\mathrm{FC}_{10} \mathrm{OH}$ molecules from the interface is also an abrupt process, however, it is a partial desorption. A large fraction of the interface remains covered by domains even above the transition temperature. ${ }^{[1]}$

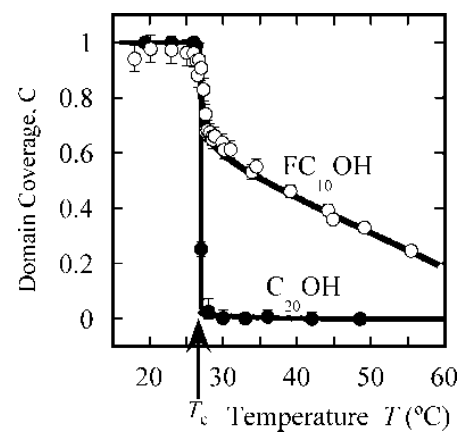

FIG. 8. Temperature dependence of domain coverage, $\mathrm{C}$ (defined as the fraction of the interface covered by surfactant domains), of the single surfactant systems: $(\bullet) \mathrm{C}_{20} \mathrm{OH}$ molecules $\left(15 \mathrm{mmol} / \mathrm{kg}\right.$ in hexane) and (O) $\mathrm{FC}_{10} \mathrm{OH}$ molecules $(5.0 \mathrm{mmol} / \mathrm{kg}$ in hexane) at the water-hexane interface. Solid lines represent the overall trend in the domain coverage variation with temperature. At the transition temperature, $\mathrm{C}_{20} \mathrm{OH}$ molecules fully desorb from the interface, unlike $\mathrm{FC}_{10} \mathrm{OH}$ molecules, which undergo partial desorption. At temperatures greater than transition temperature, there are no detectable $\mathrm{C}_{20} \mathrm{OH}$ molecules at the interface, while a significant density of $\mathrm{FC}_{10} \mathrm{OH}$ molecules is detectable even at temperatures as high as $T-T_{\mathrm{c}} \sim 30^{\circ} \mathrm{C}$.

\section{MIXED SURFACTANT SYSTEMS}

\section{Interfacial Tension Phase Diagram}

The interfacial tension phase diagram of the interface between water and a hexane solution of the two surfactants $\mathrm{C}_{20} \mathrm{OH}$ and $\mathrm{FC}_{10} \mathrm{OH}$ as a function of temperature $T\left({ }^{\circ} \mathrm{C}\right)$, total surfactant molality $m(\mathrm{mmol} / \mathrm{kg})$, and surfactant fraction of $\mathrm{FC}_{10} \mathrm{OH}, X_{2}$, is shown in Figure 9 (and was measured by Takanori Takiue, Norihiro Ikeda, and Makoto Aratono. ${ }^{[1]}$ The surfactant fraction of $\mathrm{FC}_{10} \mathrm{OH}$ is $X_{2}=0.28$ in Figure 9(a) and $X_{2}=0.25$ in Figure 9(b). Since the slope of $\gamma(T)$ determines the interfacial excess entropy $S_{a}^{\sigma}$ Equation (3), a region in the phase diagram where all the trajectories have a similar slope corresponds to a single phase and a discontinuity in the slope

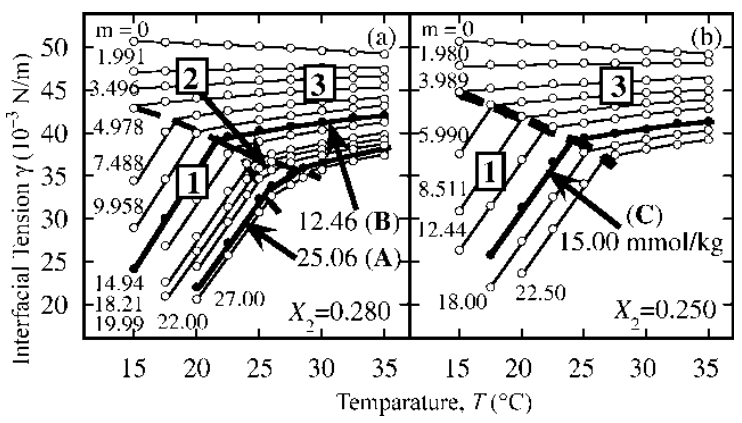

FIG. 9. Interfacial tension $(\gamma)$ of the interface between water and hexane solution of the mixed surfactant $\mathrm{C}_{20} \mathrm{OH}$ and $\mathrm{FC}_{10} \mathrm{OH}$ system as a function of temperature $\left({ }^{\circ} \mathrm{C}\right)$, total surfactant molality $m(\mathrm{mmol} / \mathrm{kg})$, and the fraction of the $\mathrm{FC}_{10} \mathrm{OH}$ surfactant molality to the total surfactant molality $X_{2}$. (a) $X_{2}=28 \%$, three phases referred to as 1,2 , and 3. (b) $X_{2}=25 \%$; two phases referred to as 1 and 3. Systems: A $(m=25.06 \mathrm{mmol} / \mathrm{kg})$, B $(m=12.46 \mathrm{mmol} / \mathrm{kg})$, and C $(m=15.00 \mathrm{mmol} / \mathrm{kg})$ represented in bold solid lines were studied using X-ray reflectivity. 
indicates a phase transition. These regions, or phases, have been labeled by the numbers 1,2 , and 3 .

Three compositions indicated in Figure 9, and denoted as systems A, B, and C, were studied with X-ray reflectivity, although we will discuss only systems $\mathrm{A}$ and $\mathrm{C}$ in this presentation. ${ }^{[11]}$ System A exhibits two discontinuities in slope (i.e., two phase transitions) between phases 1 and 2 and phases 2 and 3 (Figure 9(a)). System $C$ has one phase transition between phases 1 and 3 (Figure 9(b)). Interfacial tension measurements indicate the existence of various phases and their surface free energy, but cannot probe the molecular conformation and ordering in these phases. The tension measurements are a useful guide to allow X-ray reflectivity to efficiently probe the molecular length scale structure of the different phases and their phase transitions.

\section{X-Ray Reflectivity}

Figure 10 shows the normalized $\mathrm{X}$-ray reflectivity $\left(R / R_{\mathrm{F}}\right)$ as a function of temperature for system A. At the lowest temperature $\left(21.09^{\circ} \mathrm{C}\right)$, the $\mathrm{X}$-ray reflectivity is essentially the same as that obtained for a low-temperature single surfactant $\mathrm{C}_{20} \mathrm{OH}$ monolayer that consisted of the water-hexane interface fully covered by a liquid monolayer phase of $\mathrm{C}_{20} \mathrm{OH}$ molecules. This liquid monolayer $\mathrm{C}_{20} \mathrm{OH}$ phase is phase 1 .

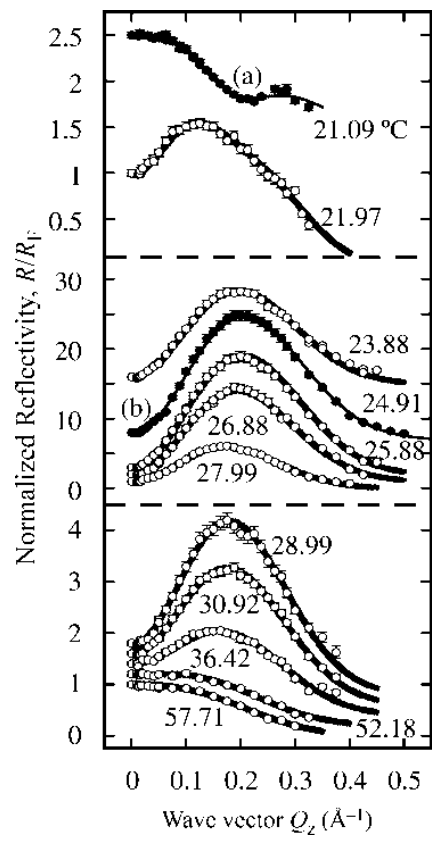

FIG. 10. Normalized X-ray reflectivity $\left(R / R_{\mathrm{F}}\right)$ as a function of wave vector transfer $Q_{z}$ and temperature $T$ for System A. (•) and (O) represents $\mathrm{X}$-ray reflectivity data and solid lines represent the fits to the X-ray reflectivity data. (a) Phase $1: \mathrm{T}=21.09^{\circ} \mathrm{C}$ curve represents a fully covered interface of a liquid monolayer $\mathrm{C}_{20} \mathrm{OH}$ phase. (b) Phase $2: \mathrm{T}=24.91^{\circ} \mathrm{C}$ curve represents a fully covered interface of a solid monolayer $\mathrm{FC}_{10} \mathrm{OH}$ phase. The remaining curves represent interfaces that consist of $\mathrm{C}_{20} \mathrm{OH}$ monolayer domains, $\mathrm{FC}_{10} \mathrm{OH}$ monolayer domains, and gas monolayer domains.
The normalized reflectivity curve at $\mathrm{T}=24.91^{\circ} \mathrm{C}$ (Figure 10) is essentially the same as that obtained for a lowtemperature single surfactant $\mathrm{FC}_{10} \mathrm{OH}$ monolayer that consisted of the water-hexane interface fully covered by a solid monolayer of $\mathrm{FC}_{10} \mathrm{OH}$ molecules. This solid monolayer $\mathrm{FC}_{10} \mathrm{OH}$ phase is phase 2 . Note that the water-hexane interface of system A at the phase transitions may have coexisting $\mathrm{C}_{20} \mathrm{OH}$ domains, $\mathrm{FC}_{10} \mathrm{OH}$ domains, and gas domains. This has been discussed in more detail elsewhere. ${ }^{[11]}$

In System $\mathrm{A}$, the interfacial phase changes from a liquid monolayer of $\mathrm{C}_{20} \mathrm{OH}$ to a solid monolayer of $\mathrm{FC}_{10} \mathrm{OH}$ with increasing temperature. This unusual phase transition will be explained below.

\section{Interfacial Domain Coverage}

Figure 11 depicts the domain coverage, $\mathrm{C}$ (fraction of the interface covered by surfactant domains), as a function of temperature for Systems A and C. A domain coverage of 1 represents a fully covered interface and a coverage of 0 represents an interface without any surfactant molecules. Below the first transition temperature, the phase 1 interface is fully covered by a liquid monolayer of $\mathrm{C}_{20} \mathrm{OH}$ molecules. At intermediate temperatures in system A only, the phase 2 interface is fully covered by a solid monolayer of $\mathrm{FC}_{10} \mathrm{OH}$ molecules. At higher temperatures, the phase 3 interface consists of domains of $\mathrm{FC}_{10} \mathrm{OH}$ molecules and gas regions.

\section{Prediction of the Mixed Surfactant System Behavior from the Two Single Surfactant Systems}

The variation of the domain coverage with temperature of the mixed surfactant systems can be explained qualitatively

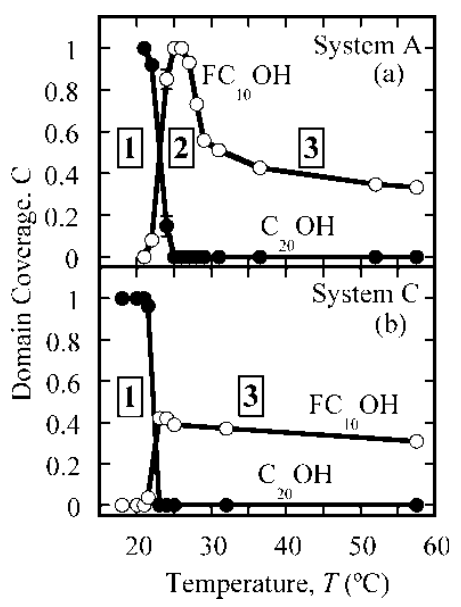

FIG. 11. Domain coverage, C (fraction of the interface covered by surfactant domains), as a function of temperature for Systems A and $\mathrm{C}$ as obtained by X-ray reflectivity: $(\bullet) \mathrm{C}_{20} \mathrm{OH}$ molecules and $(\mathrm{O}) \mathrm{FC}_{10} \mathrm{OH}$ molecules at the water-hexane interface. (a) System A: phase 1 is liquid monolayer $\mathrm{C}_{20} \mathrm{OH}$ phase, phase 2 is solid monolayer $\mathrm{FC}_{10} \mathrm{OH}$ phase; and phase 3 is a coexistence of solid $\mathrm{FC}_{10} \mathrm{OH}$ monolayer domains with gas monolayer domains. (b) System C: phases 1 and 3 have a structure similar to those in System A. 
by a weighted superposition of the two single surfactant component systems. ${ }^{[11]}$ Figure 12 shows schematically the domain coverage of the two single surfactant systems with the same concentration as that of the mixed surfactant System A (i.e., the surfactant molalities $m$ of the single surfactant $\mathrm{C}_{20} \mathrm{OH}$ and $\mathrm{FC}_{10} \mathrm{OH}$ systems are 18.04 and $7.017 \mathrm{mmol} / \mathrm{kg}$, respectively, as in the mixed system). Figure 12 illustrates two important features: (1) the phase transition temperature of the $\mathrm{C}_{20} \mathrm{OH}$ single surfactant system is $2^{\circ} \mathrm{C}$ lower than in the $\mathrm{FC}_{10} \mathrm{OH}$ single surfactant system and (2) in the single surfactant systems either $\mathrm{C}_{20} \mathrm{OH}$ or $\mathrm{FC}_{10} \mathrm{OH}$ molecules will fully cover the interface at low temperatures.

When both surfactants are present, both $\mathrm{C}_{20} \mathrm{OH}$ and $\mathrm{FC}_{10} \mathrm{OH}$ molecules cannot fully cover the interface. Our $\mathrm{X}$-ray reflectivity measurements show that $\mathrm{C}_{20} \mathrm{OH}$ molecules preferentially cover the interface at low temperatures. This can be rationalized by considering the change in interfacial excess entropy at the phase transition in the single surfactant systems. This change is essentially the free energy gain upon adsorption of the surfactant monolayer from the bulk surfactant reservoir at the transition. From the interfacial tension of the $\mathrm{C}_{20} \mathrm{OH}$ and $\mathrm{FC}_{10} \mathrm{OH}$ single surfactant systems (Figure 7), the interfacial excess entropy change at the transition, $\Delta S_{a}^{\sigma}$ (i.e., the difference between the interfacial excess entropy above and below the transition), can be determined. For the $\mathrm{C}_{20} \mathrm{OH}$ system $\Delta S_{a}^{\sigma} \approx 2 \mathrm{~mJ} /\left(\mathrm{m}^{2} \mathrm{~K}\right)$ and for $\mathrm{FC}_{10} \mathrm{OH} \Delta S_{a}^{\sigma} \approx 0.5 \mathrm{~mJ} /$ $\left(\mathrm{m}^{2} \mathrm{~K}\right){ }^{[4,5]}$ This indicates a four-fold decrease in the interfacial free energy for adsorption of $\mathrm{C}_{20} \mathrm{OH}$ molecules compared to adsorption of $\mathrm{FC}_{10} \mathrm{OH}$ molecules. Therefore, at low temperatures, a lower free energy is attained for adsorption of a monolayer of $\mathrm{C}_{20} \mathrm{OH}$.

These features allow us to predict the behavior of the mixed surfactant system by assuming that the two surfactants behave essentially independently except that the interface prefers to be occupied by $\mathrm{C}_{20} \mathrm{OH}$. So, the low-temperature phase of the mixed system consists of an interface fully covered by $\mathrm{C}_{20} \mathrm{OH}$. Upon increasing the temperature, the first transition

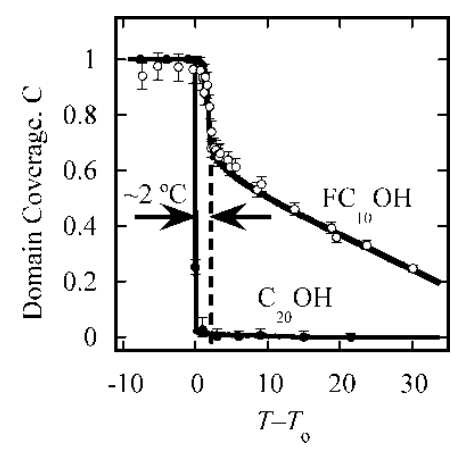

FIG. 12. Domain coverage for the $\mathrm{C}_{20} \mathrm{OH}$ and $\mathrm{FC}_{10} \mathrm{OH}$ single surfactant systems as in Figure 8, but displaced in temperature to have the same relative transition temperatures as single surfactant systems with the same concentrations as in the mixed surfactant System A.

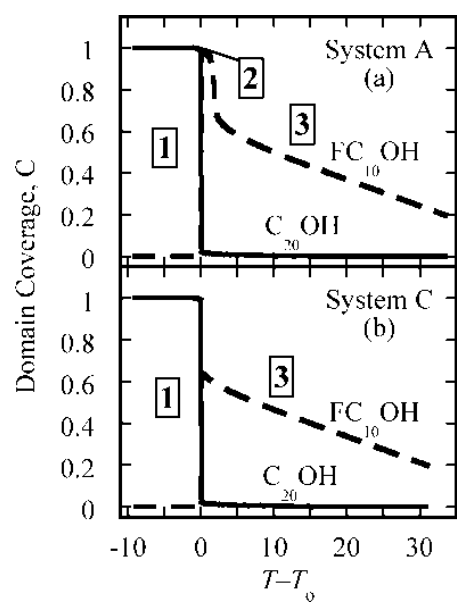

FIG. 13. The mixed surfactant system behavior of Systems A and C approximated by a weighted superposition of the two single surfactant systems; see text.

for the single component systems is the nearly complete desorption of $\mathrm{C}_{20} \mathrm{OH}$ from the interface (Figure 12). At that temperature in the mixed system $\mathrm{A}$, the interface would be available for adsorption of $\mathrm{FC}_{10} \mathrm{OH}$. As the temperature is increased further (i.e., another $2^{\circ} \mathrm{C}$; see Figure 12), the $\mathrm{FC}_{10} \mathrm{OH}$ undergoes its transition to a low density domain phase. This prediction for the mixed system A is summarized in Figure 13(a). It is qualitatively consistent with the measured behavior illustrated in Figure 11(a).

A similar prediction can be made for system C (Figure 13(b)) that is qualitatively consistent with the measurements shown in Figure 11(b). The primary difference between systems A and C is that the single surfactant systems that correspond to system $\mathrm{C}$ have a phase transition for $\mathrm{C}_{20} \mathrm{OH}$ that is $0.5^{\circ} \mathrm{C}$ above the transition for $\mathrm{FC}_{10} \mathrm{OH}$ (as opposed to it being $2^{\circ} \mathrm{C}$ below, as illustrated in Figure 12 for system $\mathrm{A}$ ). As a result, $\mathrm{FC}_{10} \mathrm{OH}$ in the mixed system never has the opportunity to fully cover the interface, and, therefore, phase 2 is absent in system $\mathrm{C}$.

\section{SUMMARY}

The recent introduction of $X$-ray reflectivity into the study of surfactant ordering and monolayer phases at the water-oil interface yields structural information on this interface that was previously unavailable. This technique probes the electron density, with sub-nanometer resolution, as a function of depth through the interface. In this presentation, at the symposium in honor of Stig Friberg, we reviewed recent X-ray reflectivity measurements combined with interfacial tension measurements that studied the interface between water and a hexane solution of the hydrocarbon alkanol $\mathrm{CH}_{3}\left(\mathrm{CH}_{2}\right)_{19} \mathrm{OH}$ and fluorocarbon alkanol $\mathrm{CF}_{3}\left(\mathrm{CF}_{2}\right)_{7}\left(\mathrm{CH}_{2}\right)_{2} \mathrm{OH}$. The surfactant molecular conformation and phase ordering are probed in the two single surfactant systems, as well as in the mixed surfactant system. In the single surfactant systems, the monolayers at the water-hexane 
interface exhibit one phase transition between a condensed monolayer phase and a dilute monolayer phase. This is in contrast with Langmuir monolayers at the water-vapor interface, which exhibit many phases. The mixed system exhibits three monolayer phases, two of which are similar to the single surfactant phases. The phases can be accessed by varying the temperature and bulk surfactant compositions. In the mixed system, a transition from a liquid monolayer to a solid monolayer occurs with increasing temperature. This unusual phase transition as well as the qualitative features of the phase diagram are predicted by an appropriate superposition of the behavior of the two single surfactant systems.

In conclusion, X-ray reflectivity is a powerful tool for the study of surfactant ordering at the water-oil interface. It can be used to probe the conformations of interfacial molecules, as well as interfacial phases and transitions between them. We have illustrated its use with simple long-chain surfactants, one hydrocarbon and one fluorocarbon surfactant, at the water-hexane interface. These measurements demonstrate the usefulness of this technique for both single surfactant and mixed surfactant systems. We anticipate that this technique can be used for the investigation of many different kinds of surfactants, including those of industrial relevance.

\section{ACKNOWLEDGMENTS}

We acknowledge conversations with Professor Dirk Morr (UIC Physics). This work was supported by the donors of The Petroleum Research Fund administered by the ACS, the UIC Campus Research Board, and the NSF Division of Materials Research. Brookhaven National Laboratory is supported by the U.S. Department of Energy.

\section{REFERENCES}

[1] Schlossman, M.L. (2002) Liquid-liquid interfaces: Studied by $\mathrm{X}$-ray and neutron scattering. Curr. Opin. Colloid Interface Sci., 7: 235.
[2] Tikhonov, A.M. and Schlossman, M.L. (2003) Surfactant and water ordering in triacontanol monolayers at the water-hexane interface. J. Phys. Chem. B, 107: 3344-3347.

[3] Li, M., Tikhonov, A., and Schlossman, M.L. (2002) An X-ray diffuse scattering study of domains in $\mathrm{F}\left(\mathrm{CF}_{2}\right)_{10}\left(\mathrm{CH}_{2}\right)_{2} \mathrm{OH}$ monolayers at the hexane-water interface. Europhys. Lett., 58: $80-86$.

[4] Tikhonov, A.M., Pingali, S.V., and Schlossman, M.L. (2004) Molecular ordering and phase transitions in alkanol monolayers at the water-hexane interface. J. Chem. Phys., 120: $11822-11838$.

[5] Zhang, Z., Mitrinovic, D.M., Williams, S.M., Huang, Z., and Schlossman, M.L. (1999) X-ray scattering from monolayers of $\mathrm{F}\left(\mathrm{CF}_{2}\right)_{10}\left(\mathrm{CH}_{2}\right)_{2} \mathrm{OH}$ at the water-(hexane solution) and watervapor interfaces. J. Chem. Phys., 110: 7421.

[6] Schlossman, M.L., Synal, D., Guan, Y., Meron, M., SheaMcCarthy, G., Huang, Z., Acero, A., Williams, S.M., Rice, S.A., and Viccaro, P.J. (1997) A synchrotron x-ray liquid surface spectrometer. Rev. Sci. Instrum., 68: 4372.

[7] Pershan, P.S. (1990) Structure of surfaces and interfaces as studied using synchrotron radiation: Liquid surfaces. Faraday Discuss., 89: 231.

[8] Tikhonov, A.M., Li, M., Mitrinovic, D.M., and Schlossman, M.L. (2001) Phase transition behavior of fluorinated monolayers at the water-hexane interface. J. Phys. Chem. B, 105: 8065.

[9] Schwickert, H., Strobl, G., and Kimmig, M. (1991) Molecular dynamics in perfluoro-n-eicosance. I: Solid phase behavior and crystal structures. J. Chem. Phys., 95: 2800.

[10] Uredat, S. and Findenegg, G.H. (1999) Domain formation in Gibbs monolayers at oil/water interfaces studied by Brewster angle microscopy. Langmuir, 15: 1108.

[11] Pingali, S.V., Takiue, T., Luo, G., Tikhonov, A.M., Ikeda, N., Aratono, M., and Schlossman, M.L. (2005) X-ray reflectivity and interfacial tension study of the structure and phase behavior of the interface between water and mixed surfactant solutions of $\mathrm{CH}_{3}\left(\mathrm{CH}_{2}\right)_{19} \mathrm{OH}$ and $\mathrm{CF}_{3}\left(\mathrm{CF}_{2}\right)_{7}\left(\mathrm{CH}_{2}\right)_{2} \mathrm{OH}$ in hexane. J. Phys. Chem. B, 109: 1210-1225. 